Jedenfalls kann heute noch weniger als vor einigen Jahren die Rede davon sein, dass den Ländern keine substanzielle Gesetzgebungsmacht verblieben wäre«.

Die Wohnungspolitik ist indessen in der Policy-Forschung der Bundesländer bislang, wenn überhaupt, nur marginal vertreten. Dies überrascht angesichts der legislativen Zuständigkeiten doch sehr (vgl. Kapitel 2.1). Eine Erklärung mag sein, dass die Wohnungspolitik zwischenzeitlich nicht mehr als eigenständiges Politikfeld, sondern als Bestandteil der Stadtentwicklungspolitik verstanden wird bzw. wurde. Dabei ist auch dieser Bereich - mit wenigen Ausnahmen wie Zimmermann (2016) - politikwissenschaftlich nur wenig erforscht. Eine andere mutmaßliche Erklärung liegt in der langjährigen politischen und gesellschaftlichen Geringschätzung dieses Politikfeldes (vgl. Kapitel 1): Folglich war auch die wissenschaftliche Aufmerksamkeit eine randständige. Sollte diese Vermutung zutreffen, so ist ab Mitte der 2o10er Jahre - dabei je die zeitlichen Verzögerungen vom Forschungsinteresse bis hin zur Publikation berücksichtigend - eine verstärkte Publikationsaktivität zu erwarten.

Die Erkenntnisse aus anderen Politikfeldern bieten aber dennoch eine wertvolle Ausgangsbasis. Erstens verhelfen sie zu einem tiefergehenden Verständnis landespolitischer Prozesse und zweitens sind insbesondere aus der Sozial- und Arbeitsmarktpolitik, aber auch aus der Wirtschafts- und Finanzpolitik, Übertragungen zum verwandten Bereich der Wohnungspolitik denkbar.

\title{
3.2 Wohnungspolitik in der Politikwissenschaft
}

Nachdem die politikfeldanalytische Literatur zu den Bundesländern als Untersuchungsebene dargestellt wurde, verengt sich das folgende UnterKapitel inhaltlich auf die Wohnungspolitik. Diesem Politikfeld ist der Bereich des barrierefreien bzw. -reduzierten Wohnraums zuzuordnen. Dabei scheint die politikwissenschaftliche Beschäftigung mit der Wohnungspolitik dem Takt der politischen Aufmerksamkeit nahtlos zu folgen: Während die 200oer und frühen 2010er Jahre eine weitgehend publizistische Abstinenz kennzeichnete, verstärkt sich in den letzten Jahren die Forschungsaktivität. ${ }^{1}$

Wie lässt sich der Forschungsstand zu »der Wohnungspolitik« kategorisieren? Denkbar wäre eine Strukturierung entlang der föderalen Untersuchungsebenen, allerdings widerspricht dieses Vorgehen der Publikations- und Denklogik vieler Autor*innen. Diese sind zumeist an einzelnen Problemen oder Policies interessiert unabhängig davon, welche politische Ebene je über Handlungskompetenzen verfügt. Vielfach lassen sich wohnungspolitische Herausforderungen ohnehin nur im Mehrebenensystem bearbeiten. Vor diesem Hintergrund wird zunächst der bestehende politikwissenschaftliche Forschungsstand zur allgemeinen Wohnungspolitik aufgezeigt. Daraufhin sind diejenigen wohnungspolitischen Debatten zu erörtern, denen die Politikwissenschaft aktuell ihre Aufmerksamkeit widmet.

1 Aufgrund der nationalstaatlich stark variierenden Wohnungsmärkte und Wohnungspolitiken beschränkt sich dieses Kapitel auf die deutsche Wohnungspolitikforschung. 
Beginnend mit der Grundlagenliteratur zur Wohnungspolitik erscheint es geboten, einen Schnitt zwischen der Zeit vor und nach dem Jahr $2006 \mathrm{zu}$ ziehen, da die damalige Föderalismusreform die institutionellen Zuständigkeiten grundlegend verändert hat. Die historisch interessierten Leser*innen finden Lektüre zur Entwicklung der Wohnungspolitik bei Heinelt/Egner (2006) und Münch (2006). Meisel (1998) analysiert den Institutionentransfer in der Wohnraumförderung nach 1989: Am Beispiel von Sachsen und Sachsen-Anhalt identifiziert er für die frühen 1990er Jahre einen »weitgehenden Transfer von Institutionen, Normen und Personal (Meisel 1998: 98). Ferner darf in einem geschichtlichen Abriss zur deutschen Wohnungspolitikforschung Everhard Holtmann nicht unerwähnt bleiben. Er hat die politischen Prozesse unter Zuhilfenahme der Strukturbruchthese von Lehmbruch analysiert (Holtmann 2000, vgl. auch Reutter 2006: 1251ff.) und kurz vor der Föderalismusreform 2006 die damalige föderale Aufgabenteilung in der Bau- und Wohnungspolitik hinterfragt:

»[...] dass es regional differenzierten Handlungsspielraum gibt, liegt auf der Hand. Wenn dies so ist, liegt die Schlussfolgerung zunächst nahe, dass eine bundeseinheitliche Normierung, wie sie für den deutschen >unitarischen Bundesstaat (Konrad Hesse) generell und auch im Sektor der Bau- und Wohnungspolitik speziell kennzeichnend ist, suboptimale Problemlösungen hervorbringt« (Holtmann 2005: 282).

Schließlich ist das einschlägige Werk »Wohnungspolitik in Deutschland « von Egner et al. (2004) unbedingt empfehlenswert, da es sowohl begrifflich und historisch die Wohnungspolitik in Deutschland aufarbeitet, als auch ihre zentralen Akteure, Institutionen und Maßnahmen untersucht.

Mit der Föderalismusreform im Jahr 2006 werden die legislativen Zuständigkeiten der Länder zwar ausgebaut (vgl. Kapitel 2.1), jedoch geht mit dieser Kompetenzverschiebung ein Rückgang der wissenschaftlichen Aufmerksamkeit für dieses nun stärker subnational organisierte Politikfeld einher: Mit Ausnahme von Lampert/Althammer (2007) - welche in ihren Ausführungen noch stark von der Zeit vor 2006 geprägt sind scheint hier tatsächlich auch in der Forschung das von von Einem (2016a) attestierte »verschlafene Jahrzehnt « zum Tragen zu kommen. Ab Mitte der $2010 e r$ Jahre wiederum ist eine erneute Publikationsaktivität zu beobachten, beginnend mit zwei interdisziplinären Sammelbänden von von Einem (2016a) und Schönig et al. (2017a). Mit Gluns (2019), Schubert (2018), Müller (2018) und Knabe/Leitner (2017) finden sich dann direkt mehrere Übersichtsarbeiten zur Wohnungspolitik in Deutschland. Während die beiden erstgenannten einen klaren politikfeldanalytischen Zugang über Akteure und Institutionen wählen, befassen sich die letztgenannten Autorinnen - der Debatte zum bezahlbaren Wohnraum folgend - mit einer Verknüpfung von Sozialpolitik und Wohnungspolitik.

Die Recherche ergab, dass es keine aktuellen Studien gibt, welche sich schwerpunktmäßig mit der Wohnungspolitik der Bundesländer auseinandersetzen. In starkem Kontrast zu diesem Vakuum genießt die kommunale Wohnungspolitik eine große Aufmerksamkeit (Naßmacher/Naßmacher 2007: 328-348; Krummacher 2011; Schönig et al. 2017b; Rink/Egner 2020a sowie die Tagungsreader von Haug et al. 2012 und der Schader Stiftung 2017).

Nach diesem chronologischen Abriss zur politikwissenschaftlichen Beschäftigung mit der Wohnungspolitik werden im Folgenden zwei wohnungspolitische Diskurse 
skizziert, die aktuell die Wohnungspolitik und auch die Wohnungspolitikforschung dominieren: Erstens die Frage der Preissteigerung in urbanen Räumen und zweitens die Schrumpfung der ländlichen Räume. Das Sichten entsprechender Schwerpunktsetzungen ist wichtig, weil sie einerseits Aufmerksamkeiten bündeln, andererseits aber auch mögliche Anknüpfungspunkte zum Untersuchungsgegenstand des barrierefreien bzw. -reduzierten Wohnraums offenlegen können.

Die Studien zur Preissteigerung und Gentrifizierung konzentrieren sich auf Ballungsräume (Breyer et al. 2018; Vollmer/Kadi 2018; Holm 2016; Schipper 2017; Schönig 2013). Hier ist die Literatur heterogen und vielfach durchwachsen von politischen Positionierungen. Neben aktivistischen Bündnissen zum bezahlbaren Wohnraum finden sich entsprechende Initiativen auch im wissenschaftlichen Raum, wenn wissenschaftliche Analysen mit politischen Forderungen vermengt werden (vgl. Die Unterzeichnenden »Für eine wirklich soziale Wohnungspolitik« 2018; Aring et al. 2016). Im Rahmen dieses Diskurses spielt die Zielgruppe der älteren Menschen durchaus eine Rolle - zusammen mit Studierenden, Alleinerziehenden oder Transleistungsbeziehenden gelten sie als eine wohnungspolitische Risikogruppe (Bundesbaublatt 2019; BBSR 2015a: 26). Über die Nennung dieser Risikogruppen und den Verweis, dass vielerorts auch »die Mitte der Gesellschaft « (Knabe/Leitner 2017: 230) durch die Wohnkosten belastet sei, geht dieser Diskurs allerdings selten hinaus und qualitative Wohnraumbedarfe zur Ausstattung der Wohnung spielen keine Rolle. Nichtsdestotrotz wäre es falsch, diesen derzeit dominierenden Strang der Wohnungspolitik deshalb außer Acht zu lassen: Aufmerksamkeit für einen Aspekt geht notwendigerweise einher mit weniger Aufmerksamkeit für alternative Aspekte. Wie viel Raum bietet der wohnungspolitische Diskurs in Zeiten der dominierenden Bezahlbarkeitsdebatte also für Subthemen wie den barrierefreien bzw. -reduzierten Wohnraum?

Der zweite prominente Diskurs befasst sich mit den ländlichen Räumen. Diese werden besonders von stadtplanerischer Seite aus bearbeitet und laufen vielfach unter dem Label »Schrumpfung« bzw. demografischer Wandel (Kühn/Liebmann 2009; Krajewski/Werring 2013; Adam et al. 2018; Kühn et al. 2017; Pallagst et al. 2017). Konkret zum Wohnen abseits der Ballungsregionen empfehlen sich Pristl (2015) und Fehlberg/Mießner (2015). Hier hilft die Unterscheidung in sogenannte entspannte Wohnungsmärkte mit einem geringen Kauf- und Mietpreisniveau - die vielfach von Fortzug geprägt sind und einem »Mietermarkt« entsprechen - und »Vermietermärkte« mit hohem Preisdruck und mehr Nachfrage als Angebot (Egner et al. 2018: 23). Die politischen und wohnungswirtschaftlichen Steuerungsmöglichkeiten sind in diesen beiden Märkten grundlegend verschieden. Während der Strang zum bezahlbaren Wohnraum in Vermietermärkten neben der politischen und öffentlichen Aufmerksamkeit auch in der politikwissenschaftlichen angelangt ist, gilt dies für den Strang zu Mietermärkten im ländlichen Raum bislang nur eingeschränkt. 